\title{
La carrière militaire de Louis Simon (1874-1914)
}

\section{Frédéric Mouriès}

\section{OpenEdition}

Journals

Édition électronique

URL : http://journals.openedition.org/ccs/826

DOI : $10.4000 /$ ccs. 826

ISSN : 2558-782X

\section{Éditeur :}

Presses universitaires de Rennes, Association des lecteurs de Claude Simon

\section{Édition imprimée}

Date de publication : 31 décembre 2011

Pagination : 167-173

ISBN : 9782354121464

ISSN : $1774-9425$

\section{Référence électronique}

Frédéric Mouriès, "La carrière militaire de Louis Simon (1874-1914) », Cahiers Claude Simon [En ligne], 7 | 2011, mis en ligne le 21 septembre 2017, consulté le 22 avril 2019. URL : http:// journals.openedition.org/ccs/826; DOI : 10.4000/ccs.826 


\section{La carrière militaire de Louis Simon (1874-1914)}

Frédéric MOURIÈS

Louis Simon est né le 27 juillet 1874 à Arbois dans le département du Jura. Il est le fils de Claude, Etienne Simon, cultivateur et de Joséphine, Françoise Chazerand. Il a trois sœurs dont deux exerceront le métier d'institutrice et un frère mort prématurément. ${ }^{1}$

Après de bonnes études scolaires que Claude Simon narre dans son roman L'Acacia (p. 62 et suiv.), Louis Simon s'oriente vers Polytechnique ; mais une mauvaise chute de cheval l'empêche de concourir (L'Acacia, p.68). Il postule alors pour le concours d'entrée de Saint-Cyr qu'il passe avec succès en 1895 , intégrant la $80^{\mathrm{e}}$ promotion, baptisée promotion Tananarive (là même où, étrange coïncidence, naîtra son fils unique Claude, en 1913). A sa sortie de Saint-Cyr, il choisit le régiment d'infanterie coloniale dans lequel il effectuera la totalité de sa carrière militaire jusqu'en $1914^{2}$.

1. Mireille Calle-Gruber, Claude Simon. Une vie à écrire, Paris, Seuil 2011, p. 25.

2. Cet article a été réalisé grâce au dossier personnel de Louis Simon conservé au service historique de la Défense à Vincennes sous la cote $4 \mathrm{Y}^{\mathrm{e}} 1290$. 


\section{La scolarité à Saint-Cyr (1895-1897)}

Des 551 élèves qui composent sa promotion, pas de noms prestigieux. Louis Simon accomplit sa scolarité sans problèmes particuliers. A sa sortie, le général Maillard, commandant l'école de Saint-Cyr, apporte une appréciation personnelle sur le sous-lieutenant Simon : "Nature franche, disciplinée, désireux de bien faire, consciencieux. $\mathrm{Ne}$ manque pas d'énergie. Bien dirigé fera un bon officier. "

\section{Tableau des notes de Louis Simon à Saint-Cyr}

Instruction générale

\begin{tabular}{|l|l|}
\hline Législation et administration 12 & Artillerie 14 \\
\hline Tactique 15 & Fortifications 15 \\
\hline Topographie 11 & Allemand 10 \\
\hline Tir 15 & Littérature 10 \\
\hline Histoire militaire 15 & Hygiène 9 \\
\hline Géographie 12 & Dessin industriel 12 \\
\hline
\end{tabular}

\section{$\underline{\text { Instruction militaire }}$}

\begin{tabular}{|l|l|}
\hline THÉORIE & PRATIQUE \\
\hline Ecole du soldat 16 & Ecole du soldat 15 \\
\hline Ecole de compagnie 16 & Ecole de compagnie 14 \\
\hline Ecole du bataillon 16 & Ecole du bataillon 14 \\
\hline Service en campagne 15 & Service en campagne 13 \\
\hline Règles de travail 15 & Tir à la cible 14 \\
\hline Service interne 16 & Equitation 12 \\
\hline Service des plans 15 & Service des plans 13 \\
\hline & Gym appliquée 18 \\
\hline & Escrime 14 \\
\hline & Exercices pratiques 13 \\
\hline
\end{tabular}


On peut observer sur son relevé de notes qu'il excelle en gymnastique, élément qui sera régulièrement remarqué dans les appréciations futures de ses supérieurs et que l'équitation n'est pas sa matière de prédilection.

Son livret militaire nous donne une description physique du tout jeune sous-officier : $1 \mathrm{~m} 73$, cheveux et sourcils châtains, yeux bleus, front moyen, nez moyen, bouche moyenne, menton moyen et visage rond. Sa santé est « bonne » et sa vue « très bonne ».

\section{La Martinique (1898-1902)}

Il embarque pour la Martinique le 30 septembre 1898. Il y gagne assez vite les galons de Lieutenant deuxième classe en 1899 puis première classe en 1902. Pourtant, les notes de ses supérieurs le décrivent comme apathique et « ayant besoin d'être guidé par un bon capitaine " même si, en 1899, on reconnaît en lui un officier intelligent et assez travailleur. En 1900, son officier supérieur constate que " le lieutenant Simon n'a fait aucun progrès ». Il renouvelle son conseil : « officier ayant besoin d'être dirigé et surveillé » et lui inflige même deux punitions graves en raison de son manque d'égards et sa mauvaise tenue envers ses camarades. Cet encadrement strict de sa hiérarchie semble avoir eu un effet favorable sur sa conduite car il est détaché à Balata pour y instruire les élèves caporaux. Cette mission ne lui vaudra aucune remarque négative de sa hiérarchie.

En 1902, il est envoyé dans un camp de chevaux pour y diriger des travaux de réparation. Là le constat est clair : «il n’y a absolument rien fait » et il a dû être rappelé et remplacé par un autre sousofficier. L'été 1902, il demande son rapatriement en métropole.

\section{Madagascar (1902-1906)}

Il part pour la colonie de Madagascar le 25 octobre 1902. Il y servira dans le premier régiment de tirailleurs malgaches. Ses officiers supérieurs notent dès 1903 un caractère susceptible et peu souple et une cohabitation plutôt brutale avec les indigènes auprès desquels il est loin de faire l'unanimité. Pourtant il possède « tout ce qu'il faut pour faire un bon officier ". 
On décide alors de lui confier plusieurs missions géodésiques. Elles se déroulent du 13 avril 1903 au 20 janvier 1904, du 18 avril 1904 au 8 octobre 1904 et enfin du $1^{\text {er }}$ mai 1905 au 5 novembre 1905. Ces missions lui vaudront les félicitations de son supérieur " un jeune et bel officier " mais surtout du gouverneur général de Madagascar, le général Gallieni qui lui adresse le 12 février 1905 une lettre personnelle de " félicitations pour les deux missions géodésiques conduites dans la région de Fort Dauphin et la basse vallée du Mangaro dont les résultats ont fait faire un progrès considérable à la cartographie de Madagascar. En menant à bonne fin ces travaux malgré des difficultés de toutes sortes dans des régions montagneuses et malsaines, vous avez fait preuve d'une grande endurance et d'aptitudes spéciales remarquables qui pourront être avantageusement utilisées dans des circonstances analogues. Je me plais à vous témoigner à ce sujet ma plus vive satisfaction. "

On retrouve louées les qualités physiques et sportives de l'officier Simon. Mais en 1906, malgré ses aptitudes à supporter le climat tropical, il tombe malade et doit être rapatrié en métropole. C'est la fin de son premier séjour malgache.

\section{La métropole (1906-1908)}

Il débarque à Marseille pour un séjour de deux semaines à l'hôpital de la ville (du 16 mai au 2 juin 1906) puis obtient un séjour de trois mois de convalescence qu'il passe à Arbois jusqu'au 3 septembre 1906.

Il est ensuite envoyé à Montpellier pour y effectuer un stage au deuxième génie pendant deux mois (de février à juin 1907). Il devient ensuite professeur adjoint de pratique équestre à Saint Maixent où il fait preuve de réelles qualités de cavalier. On semble le "spécialiser » dans l'enseignement puisqu'on le retrouve professeur de mathématiques où son excellente instruction, sa patience et sa ténacité sont remarquées et lui valent d'obtenir de très bons résultats de ses élèves.

Il revient à Perpignan en 1908 au moment de la sévère crise viticole qui entraîne de nombreuses échauffourées dans la ville. La tension persistante entraîne la réquisition du $24^{\mathrm{e}}$ Régiment d'Infanterie 
Coloniale de Louis Simon pour surveiller la préfecture et les lieux publics et rétablir la paix civile dans Perpignan.

Il profite de son passage en France pour démissionner de la Saint Cyrienne, association des anciens de St Cyr. Son fils Claude éprouvera la même indifférence envers « les Associations d'Anciens " ${ }^{3}$.

\section{Le Tonkin (1908-1909)}

Le 21 juin 1908, il embarque sur l'Armand-Béhic en direction de l'Indochine. Lorsqu'il arrive à Haïphong le 20 juillet, la situation au Tonkin est des plus tendues surtout depuis le 27 juin, date de la tentative d'empoisonnement de la garnison de Hanoi qui va entraîner une intensification de la lutte du pouvoir colonial contre les rebelles vietnamiens et les bandes chinoises.

Il rejoint la $11^{\mathrm{e}}$ compagnie du deuxième régiment des tirailleurs tonkinois basée à Loc-Binh. Il y obtient assez vite une permission de quinze jours pendant laquelle il part visiter la colonie (Langson, Hanoi, Haïphong). Mais il tombe malade et séjourne à l'hôpital de Langson du $1^{\text {er }}$ au 13 octobre 1908. Cela ne l'empêche pas d'obtenir une promotion puisque le jour de Noël, il devient capitaine.

Il commande désormais la $6^{\mathrm{e}}$ compagnie du $1^{\mathrm{er}}$ régiment des tirailleurs tonkinois qui sera impliqué dans cette lutte contre les bandits chinois dans la région de Hoangshu Phi (extrême-nord du Tonkin). ${ }^{4}$

Pourtant ses supérieurs continuent de le trouver " décidément un peu trop détaché des choses du service [...], ne s’appliquant qu'à ce qui lui plaît et recherchant par trop ses convenances personnelles [...] peut-être que, sous son nouveau grade, il apparaitra plus de zèle dans son service. Possède d'ailleurs tout ce qu'il faut pour bien faire s'il le veut " (rapport du 18 février 1909).

Cette amélioration se produit à Sontay en avril 1909 et on lui confie le commandement de la $9^{\text {e }}$ compagnie du $1^{\text {er }}$ régiment des tirailleurs tonkinois basée à Langson. Le commandant Obissier loue en Simon son intelligence, ses sérieuses qualités, même s'il a " ten-

3. Mireille Calle-Gruber, op. cit. , p.75

4. CAOM, Indochine GG 26294. 
dance à voir les choses de haut et à s'étonner des réflexions qui lui sont faites ». Toujours ce fichu caractère et ce détachement qui le poursuivent au fil des rapports.

Il est autorisé à avancer son retour de huit mois pour maladie et quitte l'Indochine le 21 novembre 1909 pour Marseille où il accoste le 20 décembre. Pendant son voyage, le colonel Le Camus envoie un dernier rapport sur le capitaine Louis Simon à sa hiérarchie le 9 décembre 1909 : « apte à servir dans un corps comprenant des indigènes, s'est bien porté pendant son séjour colonial, ne connaît aucune langue du pays, est apte aux fonctions politiques et administratives ».

\section{La métropole (1910-1912)}

Dès son arrivée en métropole, on lui accorde un congé de convalescence jusqu'au 20 mars 1910, prorogé jusqu'en juin. Il sera ensuite dirigé vers Vichy pour " $y$ jouir des eaux » jusqu’à la fin août. C'est durant cette convalescence qu'il va se marier avec Suzanne Denamiel, née le 8 novembre 1877 à Perpignan, fille de feu Alfred Denamiel, ingénieur des Ponts et Chaussées et de Louise Lacombe Saint Michel, descendante du fameux conventionnel qu'évoque Claude Simon dans Les Géorgiques. Comme pour tout militaire, il demande une autorisation de mariage à sa hiérarchie. Laquelle procède à une enquête sur la jeune fille et sa famille avec l'aide des services municipaux de la ville de Perpignan. L'autorisation est accordée, la réputation de la jeune fille et de sa famille étant excellente. De plus, elle apporte $30000 \mathrm{~F}$ de capital ainsi qu' une rente de $3000 \mathrm{~F}$ servie par sa mère. Le commandant chargé de l'enquête note que « ses espérances sont d'environ $116000 \mathrm{~F}$ ». Le mariage est célébré le 8 février 1910. Après sa convalescence, le capitaine Simon est versé au service des écuries où il retrouve un de ses domaines d'excellence, le cheval, et où il n'est guère étonnant qu'il se soit acquitté de sa tâche avec beaucoup plus de zèle que d'habitude.

\section{Madagascar (1912-1914)}

Il part pour Madagascar le 25 avril 1912. On le place dès son arrivée dans des missions d'enseignant dans lesquelles jusqu’à pré- 
sent il a bien réussi. Il occupe les fonctions de directeur des écoles de Tananarive. C'est là que naîtra son seul enfant, Claude, le 10 octobre 1913. Ce poste lui va à ravir et lui vaut quantité de superlatifs de la part du Commandant Koch : "Brillant officier. Tenue très soignée. Conduite très concrète. Tempérament calme et réfléchi. Très bon cavalier ». Il le propose même à la Légion d'Honneur.

En 1914, on retrouve " une très bonne éducation, excellent esprit. Aime les sports, les manœuvres, le cheval. Connaît bien les soldats et les traite selon leur mérite avec une tendance marquée par la bienveillance ».

Il quitte Madagascar le 13 mai 1914 pour rejoindre le 24è régiment d'infanterie coloniale à Perpignan.

Il aura donc rempli ses fonctions pendant deux ans à la satisfaction, semble-t-il, de ses supérieurs. Toutefois, un ultime rapport adressé de Tananarive, cruelle ironie, le 3 octobre 1914 (soit deux mois après sa mort au combat), est beaucoup moins élogieux. On y retrouve les reproches récurrents des rapports précédents :

"Officier prétentieux. Tempérament peu militaire. Caractère susceptible. A besoin de se surveiller et doit montrer plus de zèle dans le service pour être digne d'un avancement... S'occupe trop des questions de famille. »

\section{La guerre (1914)}

Louis Simon quitte Perpignan le 2 août 1914 pour sa dernière mission. Il fait partie du $24^{\mathrm{e}}$ régiment d'infanterie coloniale qui est chargé de repousser les attaques allemandes vers Luzy. Il est tué au combat dans la forêt de Jaulnay (Meuse) le 27 août 1914, un mois après son $40^{\mathrm{e}}$ anniversaire. ${ }^{5}$

5. Claude Simon évoque cette mort du capitaine Simon dans le chapitre 3 de son roman L'Acacia, intitulé « 27 août 1914 » $A$, p. 61-62). 